\title{
Exploration of A New-type Mode for Supporting the Elderly With Combination of Medical Resource and Nursing Resource
}

\author{
Aixiang $\mathrm{Huo}^{1}$, Peng $\mathrm{MaO}^{2}$ \\ ${ }^{1}$ Xi'an Peihua University, Xi'an 710065, China \\ ${ }^{2}$ Shaanxi Momeide Pharmaceutical Co. Ltd., Shaanxi Xi'an, 710125, China
}

Keywords: Combination of medical resource and nursing resource; New type; Mode for supporting the elderly.

\begin{abstract}
In China, population aging trend has become increasingly significant in recent years. Nowadays, the aged population has broke through 200 million. Meanwhile, due to continuous development of industrialization and urbanization, traditional family supporting mode tends to weakening. Thus, the professional nursing institution for the elderly becomes an inevitable choice. Combination of medical resource and nursing resource is one of very important modes in social nursing system and also an important measure to actively cope with population aging and improve living quality of the elderly. This paper analyzes basic features of aging in China, summarizes the meaning of combination of medical resource and nursing resource, lists main advantages of the new-type mode for supporting the elderly with combination of medical resource and nursing resource, analyzes current situation of such new-type supporting mode and proposes several countermeasures to create the new-type mode for supporting the elderly with combination of medical resource and nursing resource.
\end{abstract}

\section{Introduction}

Nowadays, economic development level improves greatly in China, and people have higher requirements for living quality. How to improve living quality of aged population is highly concerned by all sectors of society. As the pressure of population aging becomes heavier and heavier, social structure and relation between generations change greatly. Traditional supporting mode can only meet basic living needs of the elderly, but it is hard to satisfy their demand for medical treatment and health. In other words, if supporting the elderly is separated from medical treatment, it is difficult to provide good services for the elderly. Thus, it is very feasible to establish the new-type mode for supporting the elderly with combination of medical resource and nursing resource. Chinese government pays high attention to the development of elderly people supporting cause and once issued many policies. The new-type mode for supporting the elderly with combination of medical resource and nursing resource has been widely approved by most people. Hence, it is a significant strategy which can reflect social harmony, stability and fairness.

\section{Basic features of aging in China}

Compared with western developed countries, population aging in China has the following features: aging before getting rich, fast growth rate of aging population and high proportion of the disabled etc. Western developed countries usually enter the aging society when per-capita GDP reaches USD 5000-10000. For example, the proportion of population at the age of above 60 reached 12.5\% in 1950 in America, when per-capita GDP was USD 10645; the proportion of population at the age of above 60 reached $10.6 \%$ in 1970 in Japan, when per-capita GDP was USD 11579. The proportion of population at the age of above 60 reached $10.1 \%$ in 2000 in China, when per-capita GDP was only USD 3976. From 1980, in less than 20 years, population age structure in China achieved the sharp change from adult population to the aging population, while it took 80 years for Britain to achieve this process. Besides, the aged population with complete disability has exceeded 12 
million. Besides, there are numerous partially disabled people. The total number of disabled old people exceeds 40 million. Since population aging degree continues to improve and average life span further increases, the total number of disabled old people will certainly improve by a large margin. It is predicted that the total number of disabled old people will be close to 100 million by 2050 . This will bring about very heavy pressure to the society and families.

\section{Implication of combination of medical resource and nursing resource}

The appearance of the concept of combination of medical resource and nursing resource is closely related to population aging trend in China. It is an important countermeasure to practically solve the problem of separate medical treatment and nursing, and the problem of aging before getting rich. Under the new-type mode for supporting the elderly with combination of medical resource and nursing resource, independent medical treatment and health units can fully combine with nursing institutions for the elderly so as to drive effective integration of medical resource and nursing resource and achieve effective allocation of social service and social resource. "Medial resource" mainly puts particular emphasis on medical treatment and health services, and covers prevention, examination and treatment of various diseases as well as nursing; "nursing resource” mainly focuses on life, including spirit and material. The combination of medical resource and nursing resource can achieve integration of medical care and life of the elderly. This mode practically adapts physical conditions and living demands of the elderly in China, and can greatly relieve the plight where it is difficult to nurse the sick old people.

\section{Main advantages of new-type mode for supporting the elderly with combination of medical resource and nursing resource}

The combination of medical resource and nursing resource can achieve integration of medical treatment and nursing service, and utilize full connection between medical units and nursing institutions for the elderly to promote resource sharing and advantage complementation. There are mainly three modes for experimental units of the new-type mode for supporting the elderly with combination of medical resource and nursing resource: firstly, independent wards for the elderly are set in medial units; secondly, independent diagnosis, treatment and nursing institutions are set in nursing institutions for the elderly; finally, medical institutions and nursing institutions for the elderly are built together for the convenience of cooperation. The new-type mode for supporting the elderly with combination of medical resource and nursing resource has the following outstanding advantages. Firstly, national policy actively supports it, so the new-type mode has strong enforceability. Government sectors strongly support the cooperation between medial institutions and nursing institutions for the elderly, actively encourage all kinds of social forces to set up the institutions which combine medical resource and nursing resource, and drive medical units to combine nursing services for the elderly in their development. Secondly, the new-type mode can well relieve tense medical resource. At present, retention phenomenon for old patients in hospitals is very common. In fact, what the old patients badly need is not the superficial bed, but effective and high-quality medical care. Due to accelerated aging progress, more and more old patients are in sore need of professional medical services. Thus, quite heavy pressure is caused to existing medical system. The combination of medical resource and nursing resource is based on community care center and grassroots community health service station etc. It can offer high-quality health management for those old people supported in the community so that they can gain medical treatment and health service nearby. In this way, the plight of tense medical and nursing resources can be relieved well. Thirdly, basic medical care ability of nursing institutions for the elderly can improve. The setup of basic medical care department in nursing institutions for the elderly can make some old people who urgently need medical care gain services according to their health condition in the institutions which combine medical resource and nursing resource. Further, the institutions which combine medical resource and nursing resource can offer basic health service for old people in the community. Fourthly, medical service expenditure can 
be controlled and the pressure of medical health can be relieved practically. After the mode of combination of medical resource and nursing resource is implemented, current health and nursing resource can be vitalized soon so that more old patients can go out of hospitals actively, and choose home-based care or community support. In this way, medical care service expenditure can decrease, and the burden of medical insurance institutions can be relieved.

Current situation of new-type mode for supporting the elderly with combination of medical resource and nursing resource

At present, population aging trend is very significant in China. Compared with the increasing demands, the supply for the combination of medical resource and nursing resource is seriously insufficient. Currently, the number of existing nursing institutions for the elderly has exceeded 40000. However, only about $20 \%$ of institutions have the service capacity. Current situations of the mode for supporting the elderly with combination of medical resource and nursing resource are mainly reflected in the three aspects: firstly, policy guarantee is relatively insufficient. Under present policy provisions, medical institutions have no qualification to establish special wards for nursing the elderly now. Once a hospital sets up such wards, it will be at the edge of legality and illegality. Due to the lack of policy guarantee, the application for reimbursement in medical insurance department and risk resistance will be greatly limited. All kinds of medical, nursing and medical insurance policies related to the combination of medical resource and nursing resource will be restricted by financial resource. The life and medical care guarantee force for the elderly (including the disabled) is not sufficient enough, and nursing institutions for the elderly cannot offer basic health management and door-to-door care service for free. Secondly, the connection degree between medical resource and nursing resource is not high enough. Many nursing institutions for the elderly and medical institutions fail to achieve effective connection. Medical functions of numerous internal institutions are deficient. Thirdly, service capacity lags behind. In the mode for supporting the elderly with combination of medical resource and nursing resource, the elderly population mostly concerns daily health care, chronic disease and health education services etc. However, the combination of community health care services and health services is not rigorous enough. There is only care service in daytime, which cannot meet the life and medical care demands of the elderly. Similarly, due to low wage and treatment as well as limited combination of job title appraisal and employment, there are badly short of high-level managers and nursing talents in nursing institutions for the elderly. Besides, due to concept restriction, the number of young people who are willing to engage in services for the elderly decreases. Thus, existing medical service capacity cannot satisfy the demand of the elderly.

\section{Several countermeasures to create a new-type mode for supporting the elderly with combination of medical resource and nursing resource}

\section{Government sector should undertake the ultimate reasonability}

The establishment of new-type mode for supporting the elderly with combination of medical resource and nursing resource must get policy support in many aspects. Regardless of medical guarantee and nursing guarantee, relevant national sector should have the ultimate responsibility. However, for actual conditions, the state has a large gap in terms of executing the ultimate reasonability for the above two parts. Thus, it is required to continuously improve the mechanism of combining medical resource and nursing resource, and clearly specify the responsibilities of relevant national departments. Regardless of medical service and nursing service, marketization mechanism can only satisfy the people with good economic condition, and they cannot replace the government's ultimate responsibility. Hence, under the pressure of increasingly heavy population aging, the state must enhance the efforts to drive reform of medical treatment, healthy and nursing system, keep improving the expenditure scale of government sector in finance and health aspects, continuously adjust overall structure of expenditure and really form the new-type security mechanism for the elderly with combination of medical resource and nursing resource according to the actual conditions of population aging. 


\section{Specify specific operation standards of combination of medical resource and nursing resource}

The new-type mode for supporting the elderly with combination of medical resource and nursing resource is a new thing which appears in recent years. It involves extensive contents, mainly including medical treatment, nursing, entertainment and daily care etc. Because the comprehensiveness of this mode is very strong, it is not the single "medical treatment" + "nursing", but comprehensive combination of the two. Medical resource and nursing resource must cooperate with each other and give play to their active functions together. Thus, relevant government sector should overall issue various norms and standards so as to practically guide the combination of medical resource and nursing resource to achieve new development. Relevant departments can completely refer to advanced experience of western developed countries in supporting the elderly and issue a full set of professional standards with strong execution force according to actual conditions of China so as to let this new-type mode have rules to follow in the aspects of operation, evaluation and responsibility confirmation, continuously improve working ability of the institutions combining medical resource and nursing resource and achieve the ultimate purpose of overall improving living quality of the elderly in China.

\section{Form universal nursing and medical insurance expense settlement system}

Since the reform and opening-up, the first generation of immigrant workers mostly enter their old age. Quite a large number of immigrant workers will return to their hometown. One of the reasons is that they must take the annuity or apply for reimbursement of medical expense at the insurance buying place. In this way, it is difficult for them to use the annuity and medical insurance fund at the place where they live. To handle this problem well, it is required to form uniform and universal nursing and medical insurance expense settlement system so that the elderly can well handle expense reimbursement problem wherever they live and seek medical advice. As network technique becomes increasingly developed, it is technically feasible. More importantly, governments at various places must have overall consciousness, have the courage to give up vested interest under current nursing and medical mechanism and promote the work genuinely and sincerely.

Improve the treatment of medical workers in the institutions combining medical resource and nursing resource maximally

Various measures should be taken to encourage professional medical workers with the occupational qualification of physician and nurse to work at the institutions combining medical resource and nursing resource and to offer the wage and treatment of medical workers with the same level in the medical units. On this basis, the state should issue special subsidy mechanism to give certain subsidy for medical workers in the nursing institutions for the elderly. Medical treatment department and medical workers in the nursing institutions for the elderly should be under the uniform management of local National Health and Family Planning Commission. In addition, nursing institutions for the elderly should be same with medical institutions in terms of occupational qualification affirmation, professional technical post assessment and excellence appraisal. In the process of exploring combination of medical resource and nursing resource, nursing institutions for the elderly should be devoted to training nursing, medical treatment and management workers so as to further improve comprehensive quality of medical workers.

\section{Conclusion}

In conclusion, under the general background where China's economic society enters a new normal state, active construction of a new-type mode for supporting the elderly with combination of medical resource and nursing resource is a critical measure to practically solve increasingly severe aging problem. In the process of driving the new-type mode for supporting the elderly with combination of medical resource and nursing resource, China should refer to advanced experience of other countries and improve in accordance with actual conditions of China. The new-type mode for supporting the elderly with combination of medical resource and nursing resource cannot be achieved soon. It is a 
systematical project. The government should carefully make a plan, strive to introduce new concept and new mechanism in the development of the mode with combination of medical resource and nursing resource and create good conditions for $\mathrm{h}$ combination of medical resource and nursing resource from multiple aspects so as to practically solve population aging problem of China.

\section{Acknowledgments}

This paper is 2016 social sciences fund topic of Xi'an City. Topic No.: 16 S24.

\section{References}

[1] Sun Wenqian, Ding Xiancun, Study on feasibility of medical resource and nursing recourse combination mode for public hospitals - case study of Geriatric Department in Hefei Binhu Hospital, Journal of Anhui Agricultural University (Social Sciences Edition), 2013 (5).

[2] Ji Jiao, Wang Gaoling, Study on nursing institutions for the elderly with combination of medical resource and nursing recourse under coordination idea, Chinese Journal of Social Medicine, 2014 (6).

[3] Huang Jiahao, Meng Fang, Necessity, plight and strategy of nursing mode with combination of medical resource and nursing recourse, Chinese Journal of Health Policy, 2014 (6).

[4] Zhao Xiaofang, Research on nursing service mode with combination of medical resource and nursing recourse under the background of healthy aging, Lanzhou Academic Journal, 2014 (9).

[5] Li Jie, Research on problems of nursing mode with combination of medical resource and nursing recourse in Qingdao, Human Resource Development of China, 2014 (18).

[6] Chen Shao, Zhu Kehan, Li Hang, Exploration, practice and thought of combination of medical resource and nursing recourse - case study of Chengdu Chronic Disease Hospital, Sichuan Labor Guarantee, 2015 (9). 